\title{
CHD3 chromatin-remodeling factor PICKLE regulates floral transition partially via modulating $L E A F Y$ expression at the chromatin level in Arabidopsis
}

\author{
Xing $\mathrm{Fu}^{\dagger}$, Chaonan $\mathrm{Li}^{\dagger}$, Qing Liang ${ }^{\dagger}$, Yangyang Zhou, Hang He \& Liu-Min Fan* \\ State Key Laboratory of Protein and Plant Gene Research, School of Life Sciences, Peking University, Beijing 100871, China
}

Received November 12, 2015; accepted January 28, 2016; published online April 6, 2016

\begin{abstract}
PICKLE (PKL), a putative CHD3 chromatin remodeling factor, has been suggested to be involved in multiple processes in Arabidopsis. Here, we confirmed the late-flowering phenotype caused by $p k l$ mutation with $p k l$ mutants in two different ecotypes, and investigated the possible mechanisms that account for PKL regulation of flowering time. Quantitative RT-PCR and RNA-seq assays showed that expression of the $L E A F Y$ gene $(L F Y)$ and a number of LFY-regulated floral homeotic genes were down-regulated in seedlings of the $p k l$ mutants. As predicted, overexpression of $L F Y$ restored normal flowering time of $p k l$ mutants. Our results suggest that PKL may be involved in regulating flowering time via $L F Y$ expression. To uncover the underlying mechanism, ChIP-PCR using anti-PKL was performed on materials from three developmental stages of seedlings. Our results showed that PKL associated with the genomic sequences of $L F Y$, particularly at 10-day and 25-day after germination. We also showed that loss of $P K L$ affected $\mathrm{H} 3 \mathrm{~K} 27 \mathrm{me} 3$ level at the promoter of $L F Y$. Taken together, our data suggest that transcriptional regulation of $L F Y$ at the chromatin level by PKL may at least partially account for the late-flowering phenotype of $p k l$ mutants.
\end{abstract}

chromatin-remodeling factor, PICKLE, floral transition, LEAFY, Arabidopsis

Citation: $\quad$ Fu, X., Li, C., Liang, Q., Zhou, Y., He, H., and Fan, L.M. (2016). CHD3 chromatin-remodeling factor PICKLE regulates floral transition partially via modulating $L E A F Y$ expression at the chromatin level in Arabidopsis. Sci China Life Sci 59, 516-528. doi: 10.1007/s11427-016-5021-x

\section{INTRODUCTION}

Flowering is a hallmark for the transition from vegetative to reproductive phase in flowering plants. Many external and internal signals can influence the initiation of flowering, including temperature, light, and phytohormones. It is widely accepted that there are four signaling pathways that regulate flowering. They are (i) the vernalization pathway, (ii) light-dependent pathway, (ii) autonomous pathway, and (iv) gibberellin pathway (Komeda, 2004). In addition, a group of floral meristem identity genes were identified as the key factors for floral morphogenesis in the develop-

$\dagger$ Contributed equally to this work

*Corresponding author (email: lmfan@ pku.edu.cn) mental biology of plants. As one of the key floral meristem identity genes, $L E A F Y(L F Y)$ encodes a MADS box transcriptional factor, and regulates many other downstream floral meristem identity genes (Weigel et al., 1992; Winter et al., 2011). LFY is expressed in floral primordia, and its expression increases upon floral induction (Wagner et al., 1999). In addition to its role in determining floral meristem identity, increasing the copy number of wild-type $L F Y$ alleles or constitutive expression of $L F Y$ in $35 \mathrm{~S}:: L F Y$ plants also causes early flowering in Arabidopsis, indicating that $L F Y$ may also play a critical role in the initiation of flowering (Weigel and Nilsson, 1995; Blazquez et al., 1997).

In Arabidopsis, PICKLE (PKL) encodes a CHD3-like ATP-dependent chromatin remodeling factor (Ogas et al., 1997, 1999; Henderson et al., 2004; Ho et al., 2013). PKL 
was shown to regulate the transition from embryonic to vegetative development in Arabidopsis (Ogas et al., 1999). pkl mutant plants exhibit some GA-deficient phenotypes, including dark green leaves with short petioles, delayed bolting and reduced apical dominance (Henderson et al., 2004). Some of the primary roots of $p k l$ plants fail to develop normally and show some embryonic differentiation characteristics, and also accumulate triacylglycerol with a fatty acid composition which is similar to that found in seeds (Henderson et al., 2004; Rider et al., 2004). LEAFY COTYLEDON genes LEC1, LEC2 and FUS3 (FUSCA3), regulators of embryogenesis, are suppressed by PKL during germination, reflected by the fact that all the three transcripts are elevated significantly in primary roots of $p k l$ mutants (Ogas et al., 1999; Rider et al., 2003).

Further studies indicated that PKL participates in many other plant hormones signaling pathways. PKL is specifically expressed in the pericycle of primary roots and functions together with SOLITARY-ROOT (SLR)/IAA14 to control lateral root initiation (Fukaki et al., 2006). Another loss-of-function allele of $p k l$ mutant, $c k h 2$, produces green calli in response to lower concentrations of cytokinins (Furuta et al., 2011). Another study showed that $p k l$ mutants are hypersensitive to ABA treatment during germination, and that PKL suppresses $A B I 3$ and $A B I 5$ expression (Perruc et al., 2007). Moreover, abi5 mutation can rescue the ABA suppression germination phenotype of $p k l$ mutants (Perruc et al., 2007).

Apart from the role which PICKLE plays as a CHD3 chromatin-remodeling factor in regulating the transition from embryonic to vegetative development in Arabidopsis (Ogas et al., 1999), PKL was also previously implicated in promoting the initiation of flowering (Henderson et al., 2004). However, the mechanism underlying the latter process remains to be elucidated. In the present study, we showed that PKL regulates different groups of genes in three different developmental stages, and in particular, the $p k l$ mutation delays the initiation of $L F Y$ expression and the expression of many LFY-regulated genes and other flowering-related genes. Genetic analysis supports that overexpression of $L F Y$ can compromise the late-flowering phenotype of the $p k l-15$ mutant. ChIP-PCR assay shows that PKL may bind to the regulatory sequence of $L F Y$. Taken together, our work suggests that PKL may regulate flowering initiation at least partially via $L F Y$ expression at the chromatin level by binding to the regulatory sequence of the $L F Y$ gene.

\section{RESULTS}

\section{The transition from vegetative development to repro- ductive development is delayed in $p k l$ mutants}

Floral initiation is the most important change in the plant life cycle, after which the plant switches from vegetative growth to reproductive growth. A previous study showed that some GA-dependent responses were altered in $p k l$ mu- tants, including flowering time under long day conditions (Ogas et al., 1997; Henderson et al., 2004). To explore the role of PKL in the transition from vegetative to reproductive development, we determined the flowering time of wildtype and $p k l$ mutant plants under short day (SD) and long day (LD) conditions. Figure 1 shows the rosette leaf numbers of wild-type and $p k l$ mutant plants at the onset of bolting under both SD and LD conditions. Col-0 wild-type plants produced an average of 10.8 and 57.8 rosette leaves under LD and SD conditions, respectively, while pkl-1 plants produced more rosette leaves under LD and SD conditions, with the numbers 18 and 81.8, respectively. As for the Landsberg erecta (Ler) backgrounds, wild-type plants produced an average of 7.1 and 26.6 rosette leaves under LD and SD conditions, while the pkl-15 mutant plants produced 12.2 and 32.3 rosette leaves, respectively. According to the statistic results, the pkl-1 mutant had a significant delay in the transition from vegetative to reproductive development in both LD and SD conditions while $p k l-15$ displayed later floral transition under LD condition.

\section{RNA-seq supports the participation of PKL in multiple signaling pathways}

Only a limited number of flowering-related genes were
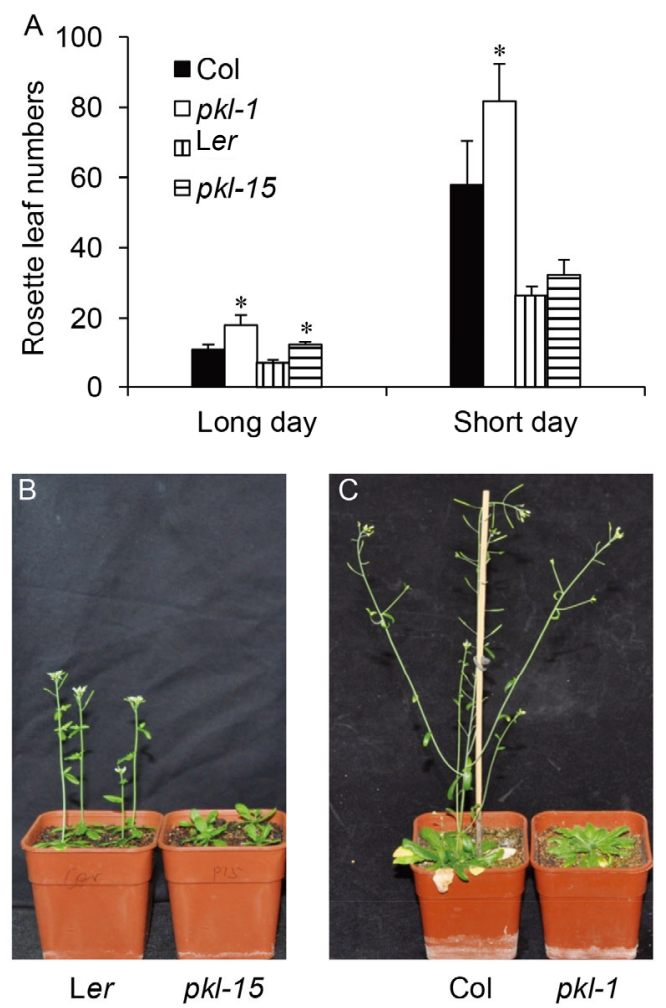

Figure $1 \mathrm{pkl}$ mutations cause delay in flowering. A, Flowering time of wild-type and $p k l$ mutants under long day and short day conditions. Flowering times were measured by total rosette leaf numbers at onset of bolting. Error bars represent the SDs. For each line, at least 30 plants were scored. *, the significant difference between the mutants and their wild-type control at $P<0.05$. B, Wild-type (Ler) and pkl-15 mutant plants grown for four weeks under LD. C, Wild-type (Col-0) and pkl-1 mutant plants grown for four weeks under LD. 
examined in semi-quantitative RT-PCR assays. As such, it is necessary to examine at a larger scale using RNA-seq, candidate genes regulated by PKL, which may account for the late-flowering phenotype in $p k l$ mutant plants. Whole seedlings at 3-day after germination, aerial parts of 10-day-old seedlings, and shoot apices of 25-day-old seedlings, of Col-0 wild-type and $p k l-1$ mutant plants, were collected in duplicates and subjected to RNA-seq analysis. The total reads passed the quality filter and the numbers of reads could be mapped back to the Arabidopsis TAIR10 genome of each sample, as shown in Table S1. For most samples, more than $95 \%$ of total reads can be uniquely mapped back to the Arabidopsis genome (Table S1 in Supporting Information). The genes with a false discovery rate (FDR) at $P$-value $<0.05$ were determined to be differentially expressed. In total, there were 1,319, 1,414 and 1,694 genes differentially regulated between wild-type and pkl-1 mutant plants at the three developmental stages, respectively, including 18, 11 and 28 loci without any annotations (Figure 2). As shown in Figure 2A, 580 or 739 genes were up- or down-regulated in 3-day old seedlings of the pkl-1 mutant; 865 or 549 genes were up- or down-regulated in 10-day old seedlings of the pkl-1 mutant; 693 or 1,001 genes were upor down-regulated in shoot apices of 25-day old seedlings of the pkl-1 mutant. Considerable numbers of differentially expressed genes were shared by each pair of developmental stages. In particular, 62 or 53 co-up-regulated or co-downregulated genes were present at all the three developmental stages (Figure 2B and C).

To further track the dynamics of the differentially expressed genes, we used hierarchical clustering to obtain clusters against all the differentially expressed genes at the three developmental stages. We used the $K$-mean clustering (Han and Kamber, 2001) method with 10 as the cluster number, and the results are shown in Figure $3 \mathrm{~A}$ and $\mathrm{B}$. Genes in clusters A-C displayed increased expression at 3-day, and reduced expression in other two developmental stages in the pkl-1 mutant. Genes in Cluster D showed reduced expression at 10-day in the pkl-1 mutant. Clusters E, $\mathrm{F}$ and $\mathrm{G}$ represent the genes with reduced expression at 25-day in the pkl-1 mutant. Clusters $\mathrm{H}$ and I include the genes that had lower expression at 3-day in the pkl-1 mutant. Cluster $\mathrm{J}$ represents the genes with increased expression at 25-day in the pkl-1 mutant. Each of clusters B, C, E, $\mathrm{F}$ and $\mathrm{G}$ includes some important flowering-related genes.

To classify the differentially expressed genes, we examined them using gene ontology (GO), which could give us a global view of functions of the differentially expressed genes. GO is a system that categorizes genes into groups or items based on their molecular function, biological process or cellular component. We mainly focused on biological processes of the differentially expressed genes.

As PKL may have different functions at different developmental stages, we examined the annotations of the differentially expressed genes at each individual developmen- tal stage. Generally, PKL may have functions in response to stimulus and lipid localization throughout seedling developmental. The stimuli mainly include stress, chemical stimulus and biotic stimulus. Remarkably, differentially expressed genes were enriched in lipid transport, secondary metabolic processes, macromolecule localization, cell wall
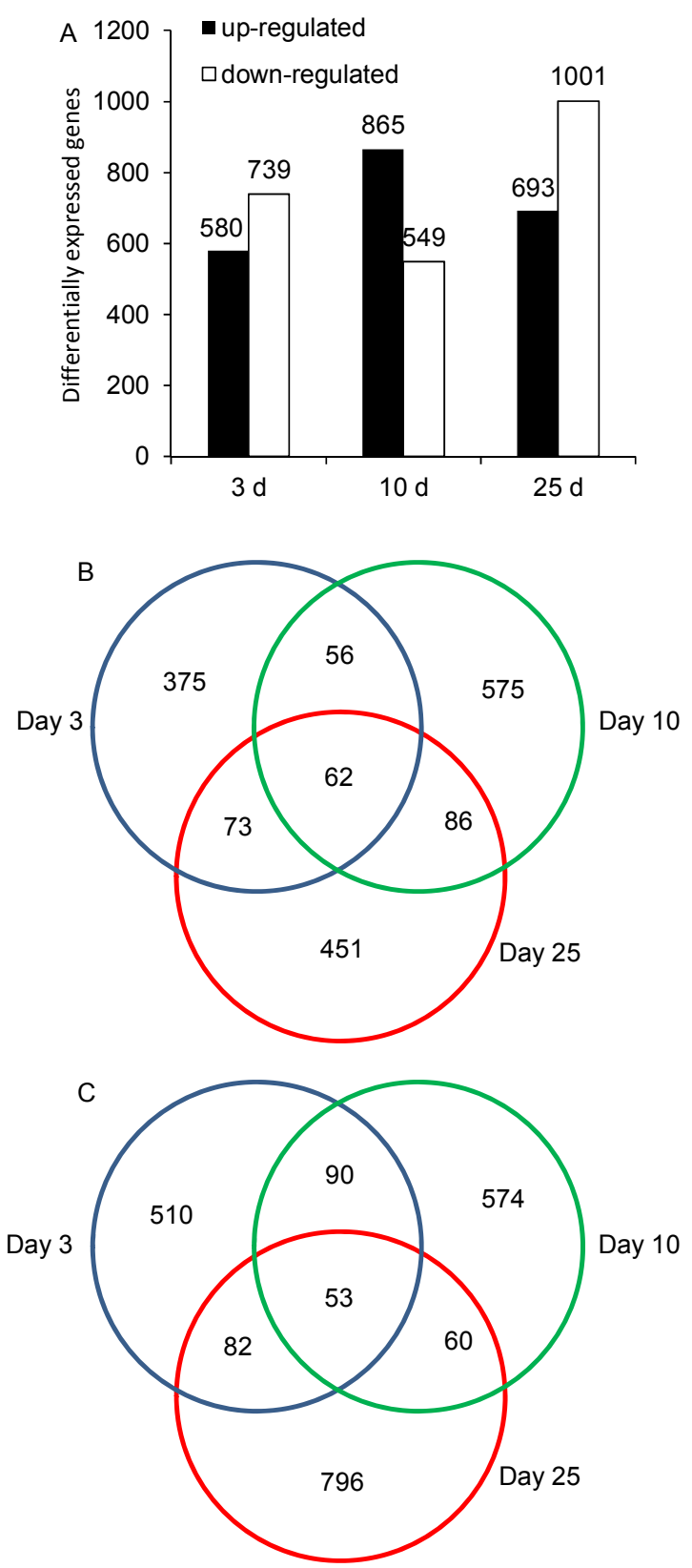

Figure 2 Differentially expressed genes at three different developmental stages. A, The numbers of up-regulated and down-regulated genes of 3, 10 and 25-day-old plant materials in the pkl-1 mutant are shown respectively. $\mathrm{B}$, Overlapping analysis of the numbers of the genes up-regulated in the pkl-1 mutant between 3, 10 and 25-day-old plant materials, presented by Venn diagram method. These data excluded all the loci without any annotations. C, Overlapping analysis of the numbers of the genes downregulated in the pkl-1 mutant between 3,10 and 25-day-old plant materials, presented by Venn diagram method. These data excluded all the loci without any annotations. 


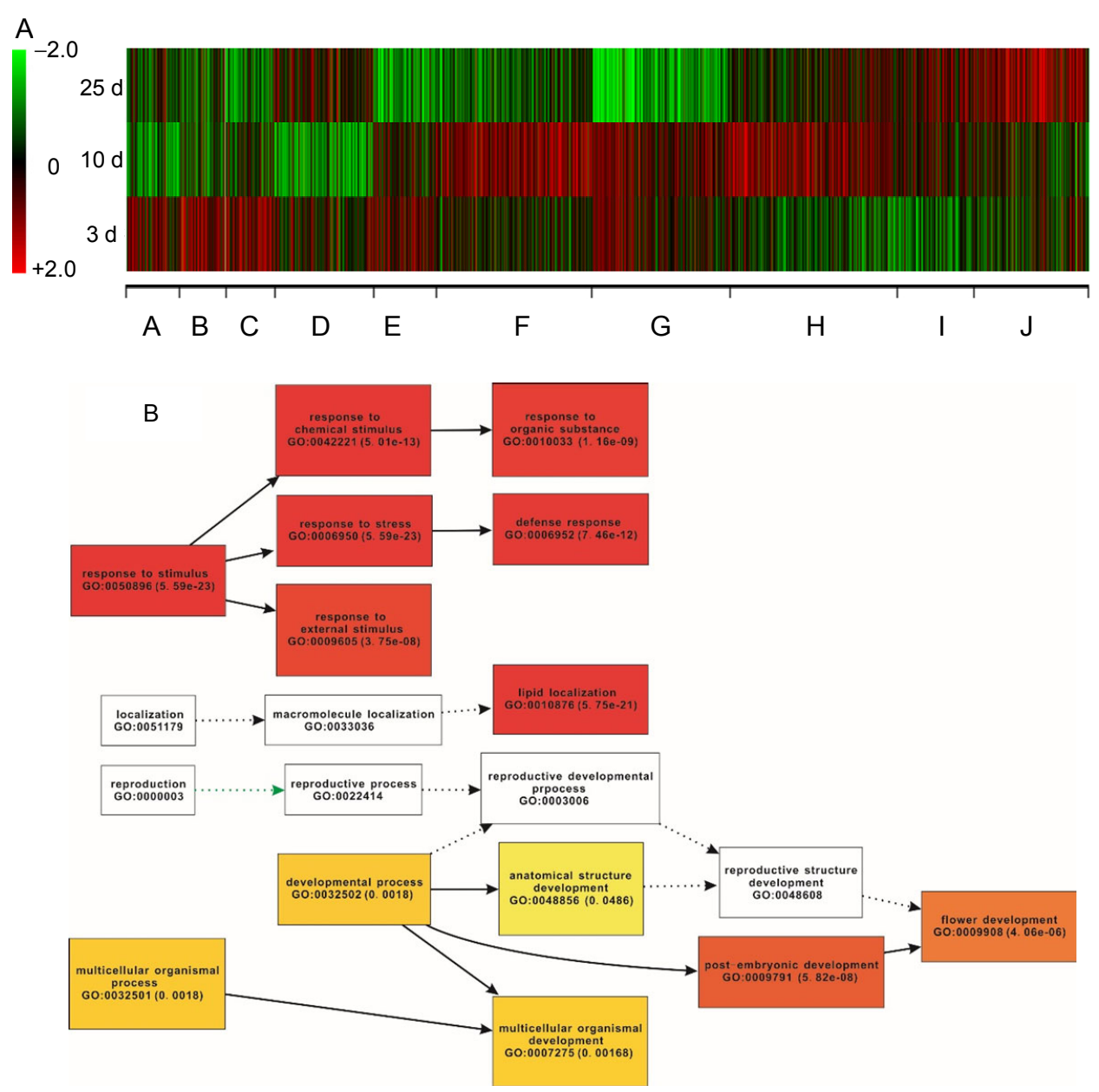

Figure 3 Clustering analysis of all differentially expressed genes. A, Heat map for profiling of all 3,031 differentially expressed genes at three developmental stages. $K$-mean clustering was performed on transcript ratios of $p k l-1$ mutant versus wild-type $\left(\log _{2} p k l-1 / \mathrm{wt}\right)$. The genes were grouped into 10 co-expression clusters A-J according to expression patterns. Red indicates higher expression in the pkl-1 mutant compared to that in wild-type, while green means lower gene expression in the $p k l-1$ mutant compared to that in wild-type. B, GO annotation of differentially expressed genes in 25-day-old shoot apex. AgriGO analysis computes GO term enrichment. The colors of the boxes stand for the degree of enrichment of the gene category; the values in the parenthesis are $P$ values, which represent the significance of the enrichment of each category.

organization, and responses to stimuli at 3-day (Figure S1 in Supporting Information). In 10-day seedlings, PKLregulated genes were specifically enriched in responses to stimuli, lipid localization and post-embryonic development (Figure S2 in Supporting Information).

GO analysis of the differentially expressed genes in shoot apices of 25-day plants showed that differentially expressed genes were enriched in responses to stimulus, lipid localization and developmental processes (Figure 3B). Furthermore, the developmental processes included post-embryonic development, flower development, and etc. (Figure 3B).

To validate the RNA-seq data, we performed quantitative RT-PCR on selected genes from the three developmental stages. As shown in Figures 4 and 5, the results of quantitative RT-PCR are in agreement with RNA-seq analysis, indicating the reliability and robustness of the RNA-seq dataset.

\section{Expression of a set of flowering-related genes is affected by mutations of $P K L$}

To uncover the mechanism(s) underlying the late-flowering phenotype of $p k l$ mutants, we focused our analysis on the flowering-related genes from the RNA-seq dataset from 25-day shoot apices. 25-day shoot apices were chosen on the basis that the onset of bolting occurs around this particular developmental stage under our experimental conditions. Expression of 41 flower development-related genes was affected in 25-day shoot apices from the pkl-1 mutant (Table S2 in Supporting Information). The expression of genes functioning in meristem identity transition was mis-regulated in the $p k l-1$ mutant, including $L F Y$ and many other floral homeotic genes. LFY is considered to be a key regulator of meristem identity transition, and also functions to activate the expression of its downstream floral homeotic genes via its binding to the specific regulatory elements close to transcription starting sites of its downstream genes, 

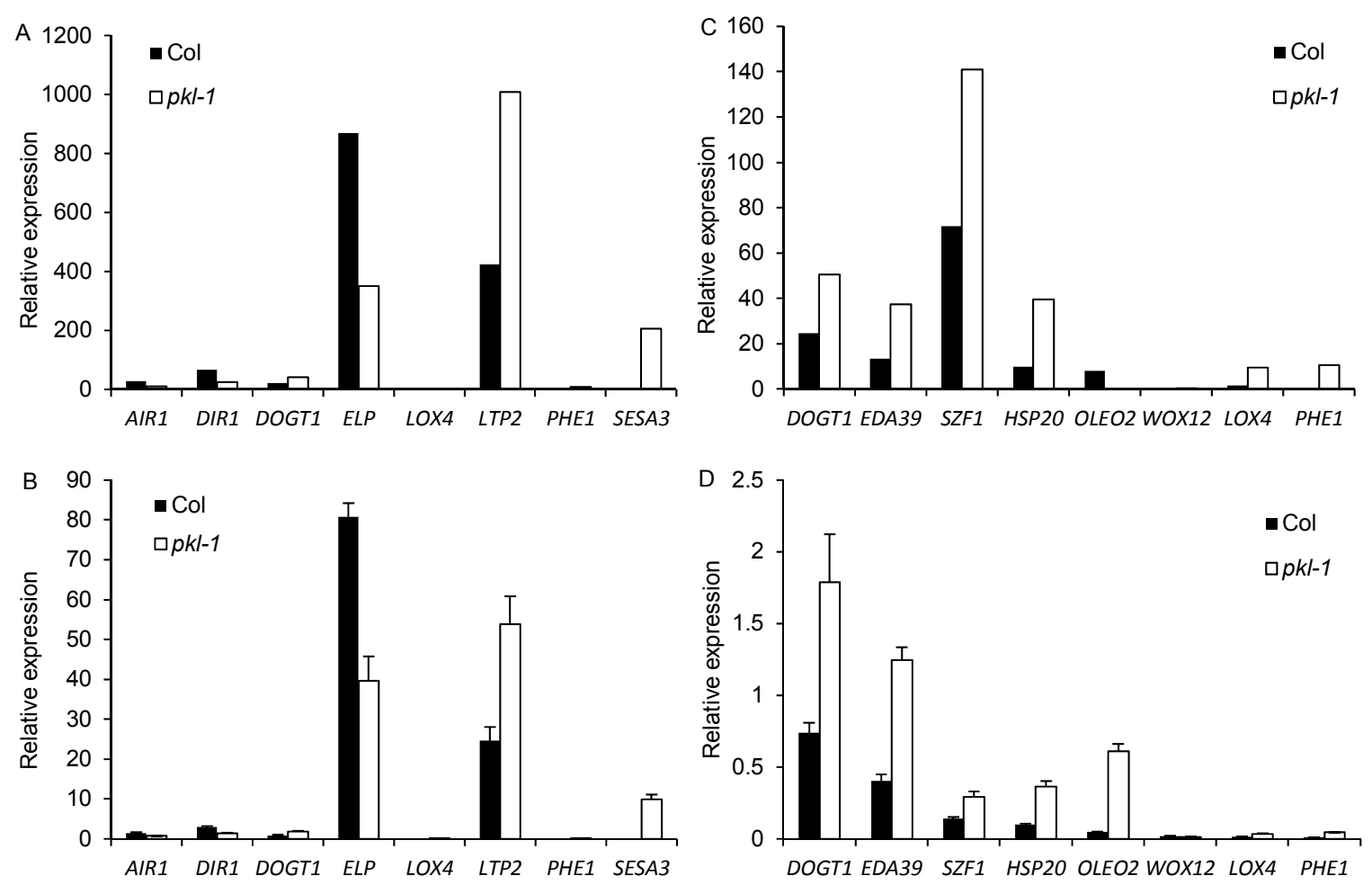

Figure 4 Lipid-related genes are regulated by PKL in 3 and 10-day-old seedlings. Expression of eight lipid-related genes in 3-day-old seedlings analyzed by RNA-seq (A) and quantitative RT-PCR (B) are shown. Expression of eight lipid-related genes in 10-day-old seedlings analyzed by RNA-seq (C) and quantitative RT-PCR (D) are shown. Relative expression in figure A and C indicates FPKM (fragments per kilobase of transcript per million mapped reads) values of the target gene in Col-0 and pkl-1 mutant samples in RNA sequencing data. All the FPKM values are calculated with Cufflinks packages. Each sample had two biological replicates. Relative expression in figure B and D indicates that the expression level of each target gene in Col-0 or pkl-1 mutant. Each sample has three biological replicates. The values are normalized relative to an internal PP2AA3 (AT1G13320) control.

thereby to direct floral organ patterning (Weigel et al., 1992; Weigel and Nilsson, 1995; Winter et al., 2011). Our RNA-seq data showed that $L F Y$ and 14 LFY-regulated downstream genes were differentially regulated in the $p k l-1$ mutant (Figure 5A, Table S3 in Supporting Information), and these genes can be mainly sorted into flowering time and floral homeotic groups (Winter et al., 2011). The RNA-seq results were confirmed by quantitative RT-PCR analysis (Figure 5B). Additionally, our data also showed that other flowering-related genes, such as JAZ1 (jasmonate-zim-domain protein 1), ARF6 (auxin response factor 6) (Nagpal et al., 2005) and SYD (splayed) (Wagner and Meyerowitz, 2002) were also differentially regulated in the pkl-1 mutant (Table S2 in Supporting Information).

To analyze the PKL-regulation of flowering-related genes in more details, we compared the expression levels of $L F Y$ and LFY downstream genes from the RNA-seq data at all of the three developmental stages between wild-type and pkl mutant plants (Figure 6). In general, the genes tested were expressed at very low levels in the mutant plants throughout the three stages although some of them, e.g., $F D, P I, S T M$, and TFLI, were expressed at higher levels in 25-day shoot apices. In wild-type plants, the expression of all of the genes tested was at low levels at 3-day post germination, but increased to a moderate level in 10-day-old seedlings, and higher in 25-day shoot apices. The expression of all of the genes tested in 25-day shoot apices were higher in the wild-type plants compared to the $p k l-1$ mutant plants.

\section{Mutations in $P K L$ delay but do not repress flower- ing-related gene expression}

As shown in Figure 5A and B, the expression of the flowering-related genes was suppressed in the $p k l-1$ mutant at 25-day after germination. From our observation, the $p k l$ mutant plants developed normal floral organs although they flowered later than the wild-type. Is expression of flowering-related genes suppressed in the $p k l$ mutant plants compared to wild-type plants? To address this, we carried out quantitative RT-PCR analysis on the flowers of the $p k l-1$ mutant and wild-type plants. As shown in Figure 5C, the flowers of the pkl-1 mutant and wild-type plants displayed comparable expression levels in terms of the genes tested. Taken together, our results suggest that the delayed flowering time associated with the $p k l$ mutation is likely via the timing of expression of flowering-related genes.

\section{Overexpression of $L F Y$ rescues the delayed flowering time phenotype of the pkl-15 mutant plants}

Both the RNA-seq and quantitative RT-PCR analyses 

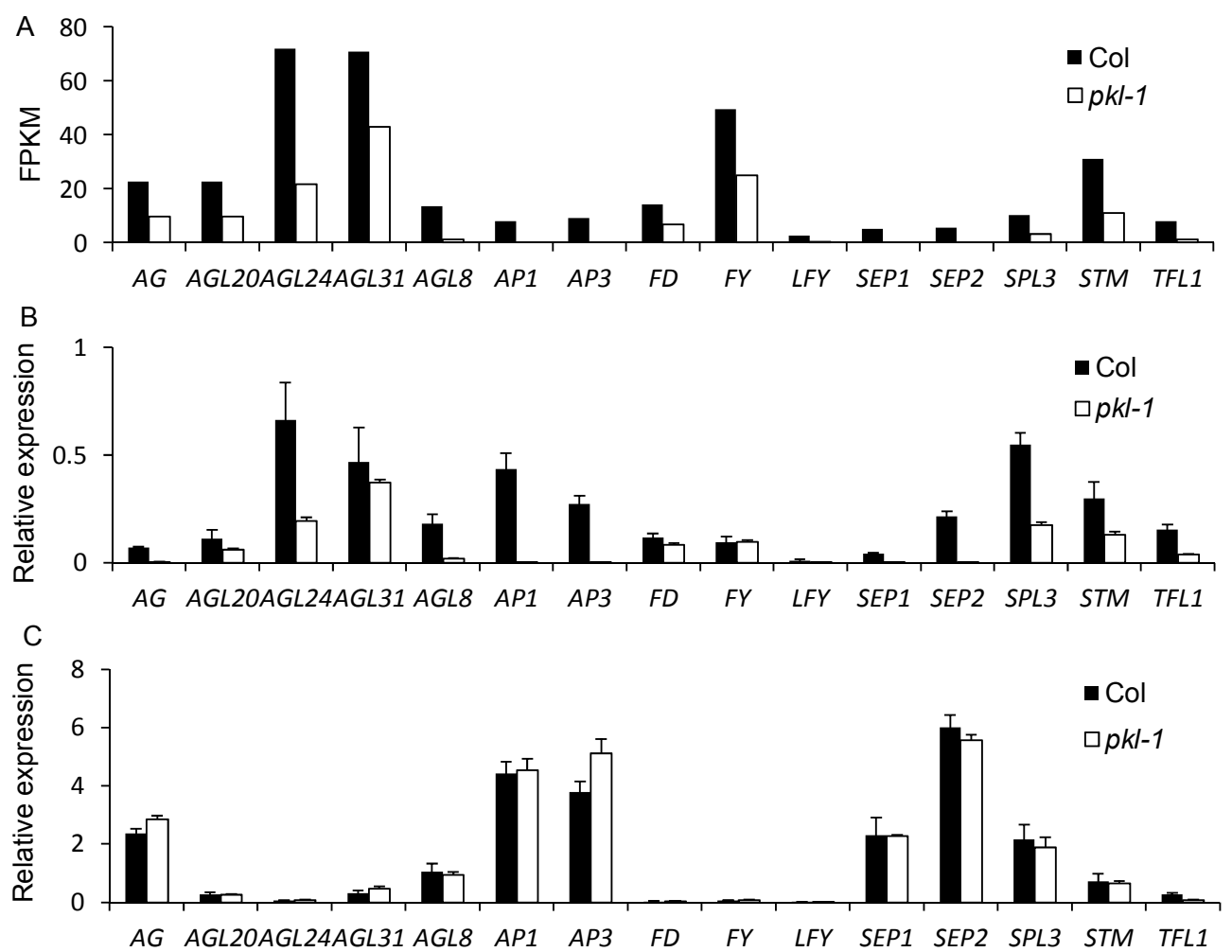

Figure 5 Flowering-related genes regulated by PKL. A, The FPKM of flowering-related genes in 25-day-old shoot apex of wild-type and pkl-1 mutant, detected by RNA-seq. B, The relative expression levels of flowering-related genes in 25-day-old shoot apex of wild-type and pkl-1 mutant, determined by quantitative RT-PCR. Each sample has three biological replicates. The values are normalized relative to an internal PP2AA3 (AT1G13320) control. C, The relative expression levels of flowering-related genes in flowers of the wild-type and pkl-1 mutant, determined by quantitative RT-PCR. Each sample has three biological replicates. The values are normalized relative to an internal PP2AA3 (AT1G13320) control.

showed that mutations in $P K L$ led to reductions in the expression of $L F Y$ and LFY downstream flowering-related genes. To test whether this reduction of gene expression was the cause for the late-flowering phenotype of $p k l \mathrm{mu}-$ tant plants, we constructed lfy-6 35S::LFY-GR pkl-15 by crossing lfy-6 35S::LFY-GR (Wagner et al., 1999) with pkl-15. Quantitative RT-PCR analyses showed that the $L F Y$ gene was over-expressed in the homozygous complemented plants (Figure 7A). Under long day conditions, by statistic analysis we observed that the over-expression of $L F Y$ can restore the normal flowering to the pkl-15 mutant (Figure $7 \mathrm{~B}$ and $\mathrm{C}$ ).

\section{PKL binds to the multiple regions of $L F Y$ and PKL facilitates $\mathrm{H} 3 \mathrm{~K} 27 \mathrm{me} 3$ enrichment at the promoter of $L F Y$ in ChIP-qPCR assay}

The homologues of PKL in animal cells, CHD3 remodelers, form the Mi-2/NuRD complex with other proteins to interact with chromatin and histones to regulate transcriptional events (Ramirez and Hagman, 2009; Xue et al., 1998). To elucidate the mechanisms of how PKL regulates gene expression, we generated polyclonal antibodies against PKL for ChIP-qPCR assay. Immunoblot analysis using anti-PKL can detect PKL protein in the wild-type but not in $p k l \mathrm{mu}-$ tant plants (Figure 8A left panel). Immunoprecipitation en- riched PKL protein instead of the nonspecific band (Figure $8 \mathrm{~A}$ right panel) that appeared in the immunoblot as shown in Figure 8A left panel. This enabled the feasibility of ChIP assay that was used to test the possible binding of PKL to the $L F Y$ locus.

To evaluate the PKL binding sites, ChIP-seq was performed on the shoot apices of Col-0 and pkl-1 mutant. Representatively, Figure $8 \mathrm{~B}$ shows that PKL binds more strongly to the genomic DNA sequences of $L F Y$ gene in Col-0 wild-type than those in the $p k l-1$ mutant. We also carried out ChIP-qPCR assay to check the binding of PKL to the genomic DNA sequences of $L F Y$ gene. Eight pairs of primers were designed to perform quantitative PCR with ChIPed DNA (Figure 8C and D). As schematically diagramed in Figure $8 \mathrm{C}$, the primers flank the fragments located in the promoter and coding regions, including the fragments 3,2 , and 1 $\mathrm{kb}$ upstream of the start codon, TSS site, two fragments at the first and second exons, and two fragments at the first and second introns. Beyond our expectation, PKL enriched all of the fragments described above in Ler wild-type material compared to those in the pkl-15 mutant (Figure 8D). However, no apparent enrichment was achieved for the ACT2 (ACTIN2) locus (Figure 8D). These ChIP-qPCR results indicate that PKL can bind to the multiple regions of the genomic sequences of the $L F Y$ gene. 

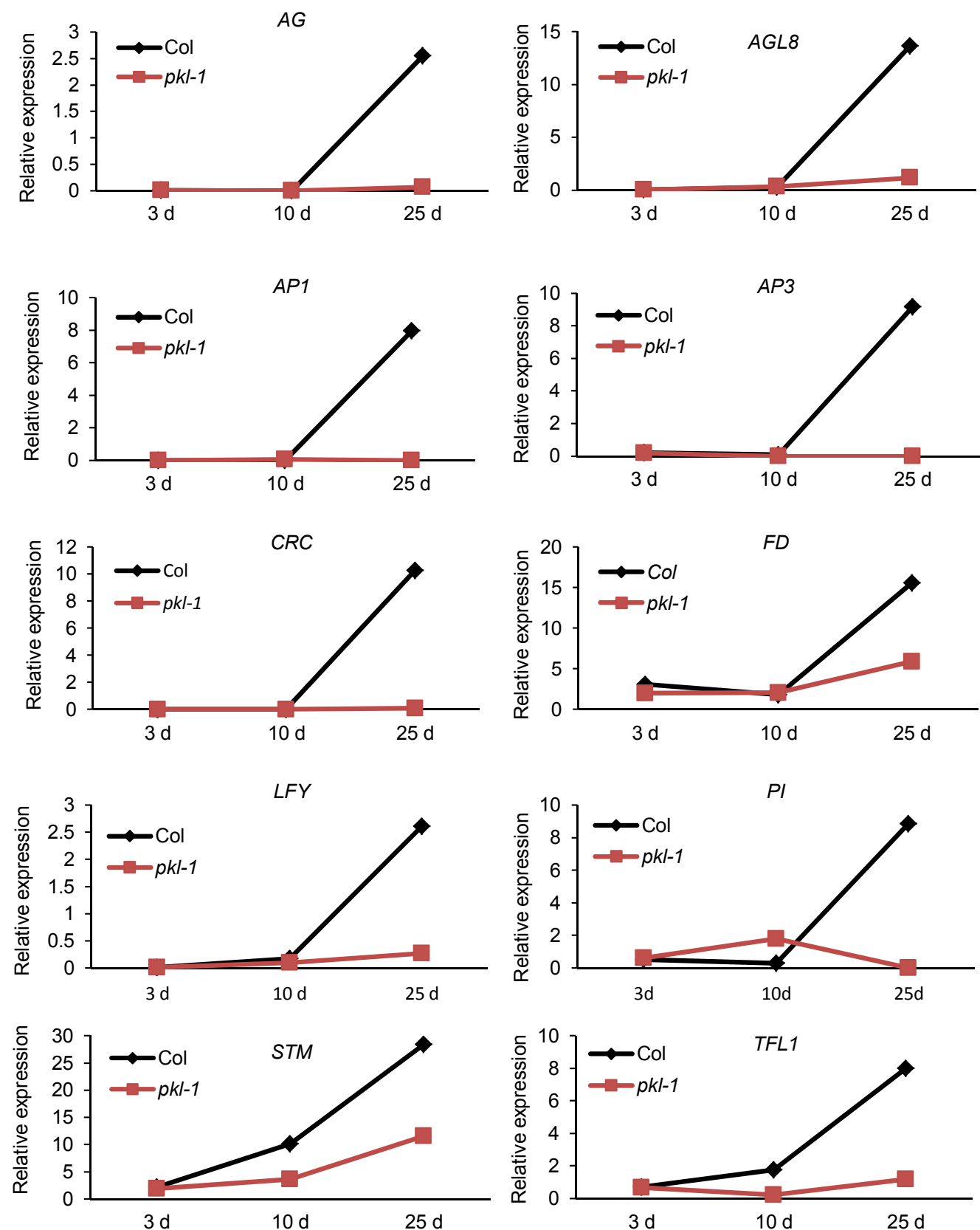

Figure 6 Dynamics of expression of PKL-regulated flowering-related genes associated with developmental stages. Expression of flowering-related genes at three development stages of wild-type and pkl-1 mutant was determined by RNA-seq assay. Relative expression indicates FPKMs of the target genes in Col-0 and pkl-1 mutant samples in RNA-seq data. All the FPKM values are calculated with Cufflinks packages. Each sample had two biological replicates.

A previous study reported that deposition of $\mathrm{H} 3 \mathrm{~K} 27 \mathrm{me} 3$ at the genes assayed was consistently reduced in pkl plants. Those genes included ACT7 (ACTIN7), MULE (mutator-like element), LEC1, LEC2, FUS3, AtBMIIC, PHE1 (pheres 1), Atlg78750, CLF (curly leaf), MEA (medea), AGL8 (agamous-like 8), SPL5 (squamosa promoter binding protein-like 5) and SOCl (suppressor of overexpression of co 1). To evaluate whether the $p k l$ mutation affects the deposition of $\mathrm{H} 3 \mathrm{~K} 27 \mathrm{me} 3$ at the promoter of $L F Y$, we performed ChIP-qPCR with anti-H3K27me3. We observed that deposition of $\mathrm{H} 3 \mathrm{~K} 27 \mathrm{me} 3$ at the promoter of $L F Y$ was signifi- cantly reduced in the $p k l-1$ mutant. The results were the same for the genes such as ACT7, MULE, LEC2, FLC and PHE1 despite the different degree of PKL effect on H3K27me3 enrichment (Figure 9).

\section{DISCUSSION}

The function of LFY is not limited to determining floral organ development

$L F Y$ is considered to be one of the key floral meristem iden- 

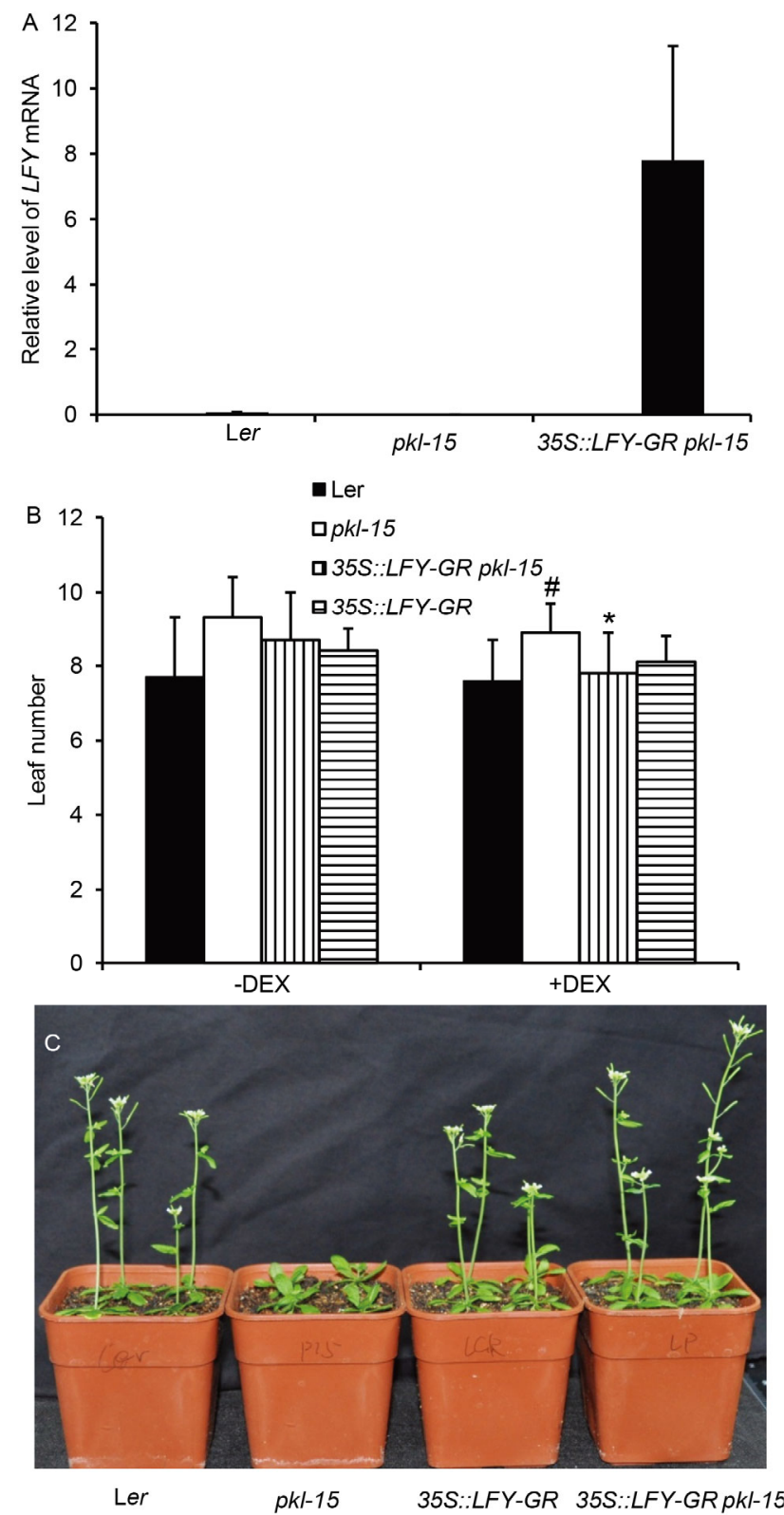

Figure 7 Constitutive expression of LFY restores normal flowering in the pkl-15 mutant. A, Expression level of $L F Y$ in transgenic plants. RNA samples were extracted from 10-day-old seedlings and the expression level was tested by RT-qPCR. AT1G13320 was used as native control. Error bars represent standard deviations. B, Flowering time is presented as the number of leaves. Plants were mock-treated with $0.1 \%$ ethanol or treated with $5 \mu \mathrm{mol} \mathrm{L}{ }^{-1}$ dexamethasone as described in the MATERIALS AND METHODS. Rosette leaf numbers were counted ( $n \geqslant 12$ plants). Error bars represent standard deviations. *, $P<0.05$ compared to $p k l-15$. \#, $P<0.05$ compared to Ler. C, The plants shown from left to right are Ler, $35 S:: L F Y-G R$ and $35 S: \because L F Y-G R$ pkl-15, that were treated with $5 \mu \mathrm{mol} \mathrm{L}^{-1}$ dexamethasone and grown for further four weeks under long day condition.

tity genes, and regulates many other downstream floral meristem identity genes (Weigel et al., 1992; Winter et al., 2011). In addition to its role in determining floral meristem identity, $L F Y$ has also been shown to regulate flowering time in Arabidopsis (Blazquez et al., 1997; Weigel and

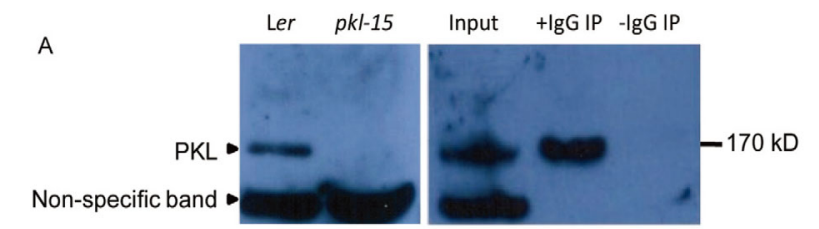

B
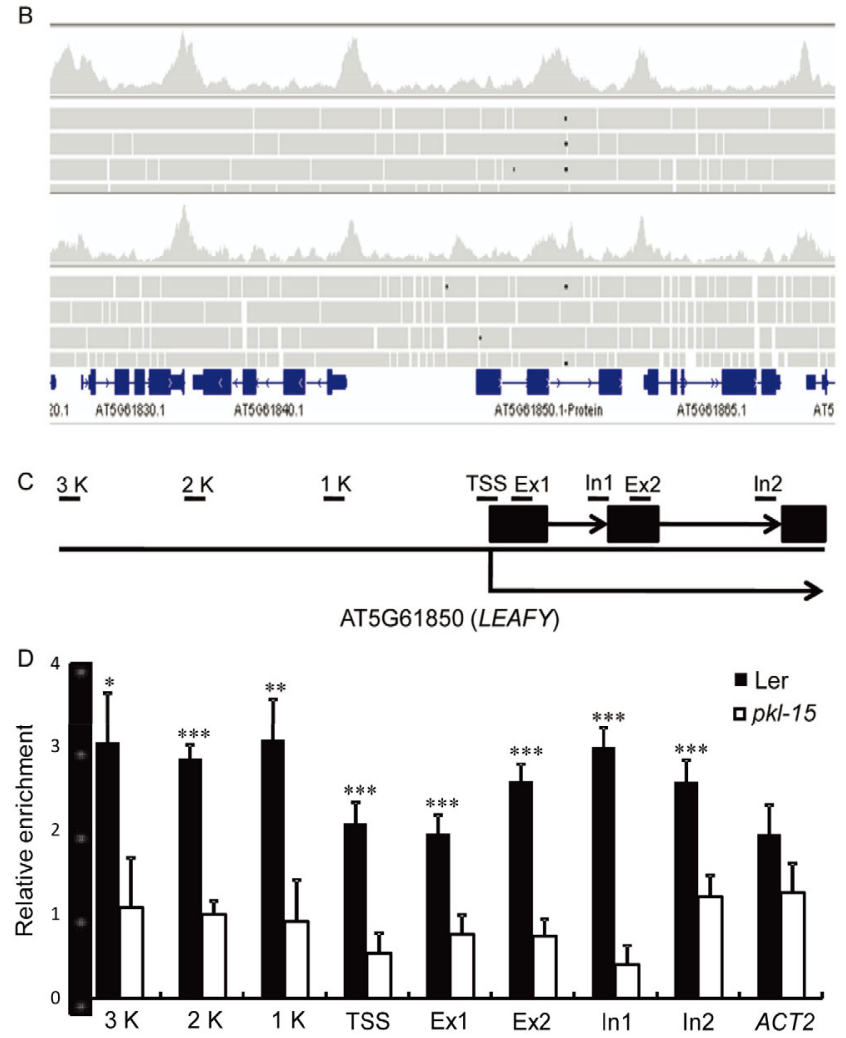

Figure 8 ChIP-seq and ChIP-qPCR analyses of PKL association with the LFY genomic sequences at shoot apex. A, Polyclonal PKL-antibody recognizes PKL protein (left panel), and specifically immunoprecipitates PKL protein from total lysates of seedlings (right panel). Total proteins of seedlings of Ler or pkl-15 mutant plants grown for $12 \mathrm{~d}$ on MS media under 16 $\mathrm{h}$ light/8 h dark condition, were extracted before western blot analysis or immunoprecipitation using polyclonal antibody against PKL. In immunoprecipitation assay, Input represents the sample of total lysate, +IgG IP and -IgG IP indicate that PKL antibody was added or not in immunoprecipitation. B, ChIP-seq presentation of PKL antibody immunoprecipitated DNA fragments flanking $L F Y$ gene. Col-0 and $p k l-1$ seedlings were grown on MS medium for $25 \mathrm{~d}$ under long day condition ( $16 \mathrm{~h}$ light $/ 8 \mathrm{~h}$ dark) at $22^{\circ} \mathrm{C}$. The shoot apices were collected for genomic DNA extraction before ChIP-seq. C, Schematic diagrams of fragments on the $L F Y$ genomic sequence used in the ChIP-qPCR assay. Black bars indicate the position of eight fragments. $3 \mathrm{~K}$, a fragment $3,000 \mathrm{bp}$ upstream of the TSS; $2 \mathrm{~K}$, a fragment 2,000 bp upstream of the TSS; $1 \mathrm{~K}$, a fragment 1,000 bp upstream of the TSS; TSS, a fragment including transcriptional start site; Ex1, a fragment in the exon1; E2, a fragment in the exon2; In1, a fragment in the intron1; In2, a fragment in the intron2. D, ChIP-qPCR assay of PKL enrichment on different locations of the $L F Y$ genomic sequence. Ler and pkl-15 seedlings were grown on MS medium for $12 \mathrm{~d}$ under long day condition ( $16 \mathrm{~h} \mathrm{light} / 8 \mathrm{~h}$ dark) at $22^{\circ} \mathrm{C}$. Each experiment had three biological replicates, and each biological replicate included three technical replicates. Standard derivations were calculated from three technical replicates. The genomic locations of different fragments used in this assay are shown in (A). ACT2 served as negative controls. The error bar indicates \pm SD. Asterisks indicate significant differences between Ler and $p k l-15$ at $P<0.05$ (*), $P<0.02(* *)$ or $P<0.01(* * *)$ using Student's $t$ test. 


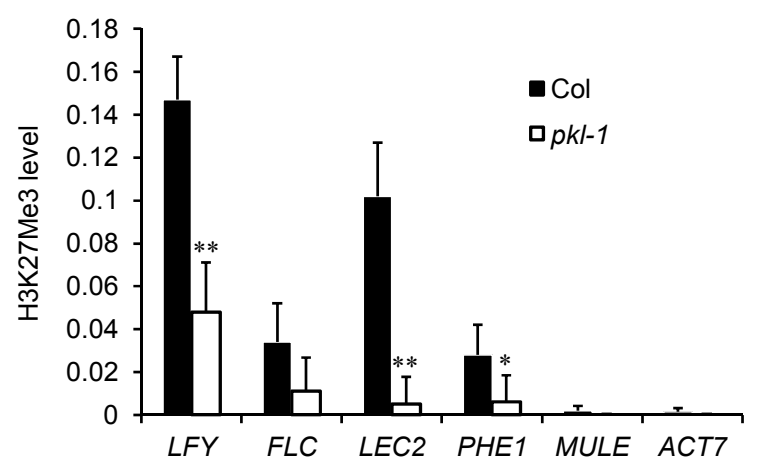

Figure 9 ChIP-qPCR analyses of H3K27me3 levels at the $L F Y$ genomic sequences. ChIP was carried out with rosette leaves of 35-d-old wild-type and pkl-1 plants using antibody (Upstate) to H3K27me3 using cross-linked DNA from wild-type (Col-0) and pkl-1 plants. All data are mean \pm SD of three biological replicates. Asterisks denote significant differences between Col-0 and $p k l-1$ at $P<0.05$ (*), $P<0.01$ (**) using Student's $t$ test.

\section{Nilsson, 1995).}

A previous study has demonstrated that flower initiation is promoted by a combination of $L F Y$ expression and competence to respond to $L F Y$ activity (Weigel and Nilsson, 1995). Another work by Blazquez et al. extended this observation and showed that the time to flowering is critically affected by levels of $L F Y$ expression in its normal pattern, as determined with plants that carry one, two, three or four copies of wild-type $L F Y$ (Blazquez et al., 1997). Therefore, $L F Y$ has been considered to function in regulating both flowering-time and flower-meristem-identity (Blazquez et al., 1997). FLO, the $L F Y$ ortholog in Antirrhinum, affects development of both individual flowers and of full inflorescence traits (Bradley et al., 1996), which is another example for the dual roles of $L F Y$.

In the present study, our observations showed that $L F Y$ may mediate PKL-modulated vegetative to reproductive transition. PKL regulation of vegetative to reproductive transition was firstly observed by Ogas et al. (Ogas et al., 1997). We carefully examined the number of rosette leaves at the onset of bolting of $p k l$ mutants and their corresponding wild-types, Col-0 and Ler under both short-day and long-day conditions. Our observations further confirmed the results of Ogas et al. (Ogas et al., 1997). A combination of RNA-seq analysis, genetic dissection and ChIP-qPCR analysis suggests that the late-flowering phenotype of $p k l \mathrm{mu}-$ tants may be ascribed to a reduction in $L F Y$ expression. Our observations support the notion that $L F Y$ is a both flowering-time and flower-meristem-identity gene, and plays dual roles both in floral organ development and the transition from vegetative to reproductive development.

\section{PKL regulates the transition from vegetative to repro- ductive development but does not determine flowering organ identities}

It has been known that PKL promotes the transition from embryonic to vegetative development (Ogas et al., 1999). $p k l$ mutants tend to maintain the embryonic state as evidenced by the observations that $p k l$ mutants are defective in repressing embryonic differentiation characteristics after germination (Ogas et al., 1997), which include expression of genes for seed storage proteins and accumulation of large amounts of neutral lipids (Ogas et al., 1997; Henderson et al., 2004; Rider et al., 2003, 2004).

As a putative chromatin remodeling factor, PKL may regulate many important developmental events. In the present study, we further show that PKL is also involved in regulating the transition from vegetative to reproductive development. Therefore, it is likely that PKL may be a global regulator of plant developmental programming. However, similar to the observation that $p k l$ mutants have the capacity to germinate (albeit after a delay), the delayed flowering in $\mathrm{pkl}$ mutants did not affect normal floral organ development. This suggests that PKL is not absolutely required for but might be a regulator of either transition from embryonic to vegetative development or transition from vegetative to reproductive development.

Whether PKL-regulated programming of developmental transitions is controlled by its regulation of gene expression is an open question. Our RNA-seq and qRT-PCR analyses show that PKL is essential for proper temporal expression of $L F Y$ and other flowering related genes. Additionally, our assays showed comparable expression of $L F Y$ and other flowering-related genes in flowers of $p k l$ mutants relative to their wild-type plants (Figure 6). Together, these data suggest that PKL may be an important regulator of floral transition, and also explain why $p k l$ mutations do not bring about any floral defects.

\section{How does PKL regulate $L F Y$ expression?}

In the present study, we show that PKL may regulate the expression of $L F Y$ and LFY-regulated downstream genes. But it remains unclear how PKL regulates their expression. As previously reported, GA pathway is one of the important pathways in regulating flowering time (Blazquez et al., 1998). Thus, it remains to be answered whether GA pathway is involved in PKL regulation of flowering time. It has been shown that the $p k l$ mutant has reduced GA content and exogenous GA application somehow rescues the delay in flowering time caused by the $p k l$ mutation (Saleh et al., 2008), indicating that GA might partially mediate $p k l$ mutation caused delay in flowering time.

PKL is a putative chromatin remodeling factor, which contains a chromo domain, a SNF2-related helicase/ATPase domain, a DNA binding domain and a PHD zinc finger (Ogas et al., 1999). These domains are typically found in chromatin-remodeling factors. Our ChIP-qPCR assays demonstrate that PKL associates with DNA sequences of the $L F Y$ gene. This points to the possibility that PKL regulation of $L F Y$ expression may be executed by the binding of PKL with $L F Y$ sequences to facilitate the activation of the $L F Y$ gene. PKL has been previously proposed to be a tran- 
scriptional activator of gene expression via direct binding to gene promoter, based on the results that direct PKL binding can only be detected to the down-regulated genes in $p k l$ mutants (Aichinger et al., 2009). However, the study by Zhang et al. did not support that PKL functions as a transcriptional activator, based on their results that the $p k l \mathrm{mu}-$ tation reduced $\mathrm{H} 3 \mathrm{~K} 27 \mathrm{me} 3$ levels and increased expression for LEC1, FUS3 and several other loci (Zhang et al., 2008). The role of PKL as a transcriptional repressor was also supported by the fact that PKL preferentially associated with H3K27me3-enriched genes and actively transcribed genes, and preferentially and specifically promoted H3K27me3 enrichment of examined PKL-dependent and nondependent genes (Zhang et al., 2012). However, in the case of the $L F Y$ gene, it is plausible that PKL may be an activator of the $L F Y$ gene expression via binding to this gene although $\mathrm{H} 3 \mathrm{~K} 27 \mathrm{me} 3$ enrichment at the promoter of $L F Y$ was promoted by the presence of PKL (Figure 9, the present study). A similar case was reported by Zhang et al., where the transcription of $A G L 8$ was upregulated but H3K27me3 enrichment at this gene was promoted by PKL (Zhang et al., 2012). Therefore, we proposed that PKL can act as either a transcriptional activator or repressor, which depends on the different individual gene in a specific case.

Regarding the specificity of PKL association, PKL not only binds to the promoter regions but also binds to other regions of the $L F Y$ gene in our ChIP-qPCR. Thus, the binding of PKL to the $L F Y$ gene is not strictly DNA sequence specific. In our ChIP assay, PKL can also weakly bind to the DNA sequence of a control gene ACT2. Therefore, the binding activity of PKL is even not specific to its target genes. Similarly, it has also been previously found that PKL was present at the promoters of either H3K27me3 enriched genes, or actively transcribed genes that were ubiquitously expressed and PKL-independent genes such as ACT7 and $U B Q 10$ (polyubiquitin 10) (Zhang et al., 2012; Harmeyer et al., 2015). Based on these data, it was even thought that PKL facilitates a common chromatin remodeling process that is not restricted to specific regions of the genome (Zhang et al., 2012). As for the molecular mechanism for the PKL regulation of $L F Y$ expression, it is likely that the $L F Y$ gene may be one of the genes which are controlled by PKL via global chromatin modification. Given that expression of other two floral regulator AGL8 (Ferrandiz et al., 2000) and AGL24 (Michaels et al., 2003) were reduced in pkl mutants, and H3K27me3- enrichment at AGL8 was reduced in $p k l$ mutants, the late-flowering phenotype of $p k l$ mutants may also in part be due to reduced expression of AGL8 (Zhang et al., 2012). In this respect, it is pertinent to make a conclusion that $L F Y$ may partly mediate the PKL regulation of flowering time control via chromatin modification. Interestingly, AGL24 and SOC1 regulate each other and act together to activate $L F Y$ transcription (Liu et al., 2008; Lee et al., 2008) while both $A G L 8 / F U L$ and $L F Y$ are independent downstream targets of SPL3 in flowering time and meristem identity control (Yamaguchi et al., 2009). Both SOC1 and $L F Y$ are direct targets of AGL8/FUL that binds to a region $2.2 \mathrm{~kb}$ upstream to the ATG codon of the LFY gene (Balanza et al., 2014), overlapping with a previously identified region that is also bound by SOC1 (Lee et al., 2008). In this context of relationship with LFY, AGL8 and AGL24 may likely be involved in PKL-regulated flowering time control in spite of being the direct targets of PKL in addition to the involvement of LFY.

\section{MATERIALS AND METHODS}

\section{Plant materials and growth conditions}

Plants of the Landsberg erecta (Ler) and Col-0 wild-types accessions and mutants in their backgrounds were used in this work. The following mutants were used: pkl-1 (Col-0 background) (Henderson et al., 2004), pkl-15 (Ler background) (Eshed et al., 1999; Ori et al., 2000). lfy-6 35S: $L F Y$-GR was a generous gift from Dr. Doris Wagner. $p k l-15$ 35S:LFY-GR was generated by crossing $p k l-15$ and lfy-6 35S:LFY-GR. For regular seedling development, seeds were stratified at $4{ }^{\circ} \mathrm{C}$ in darkness for four days, and then transferred onto and grown on half-strength Murashige and Skoog agar plates at $22^{\circ} \mathrm{C}$ under long day $(16 \mathrm{~h}$ light $/ 8 \mathrm{~h}$ darkness) or short day condition ( $8 \mathrm{~h}$ light/16 h darkness).

\section{Determination of flowering time}

To determine the flowering times of plants, seeds were stratified at $4^{\circ} \mathrm{C}$ for four days in the dark before seeded and grown for 10 days on $1 / 2$ strength MS agar plates at $22^{\circ} \mathrm{C}$ under long day (18 h light/6 h darkness) or short day condition (6 $\mathrm{h}$ light/18 $\mathrm{h}$ darkness). The seedlings were then transferred to and grown in plastic pots containing a mixture of substrate and verminculite $(2: 1)$. For dexamethasone treatment, seedlings were treated twice, at one week and two weeks old, with $5 \mu \mathrm{mol} \mathrm{L} \mathrm{L}^{-1}$ dexamethasone or $0.1 \%$ ethanol (as mock).

The number of leaves was recorded as an adequate measurement of flowering time. Numbers of rosette leaves and cauline leaves at onset of bolting were counted, respectively.

\section{Antibody preparation}

Anti-PKL antiserum was generated from rabbits immunized with the recombinant C-terminal (1199-1382 aa) region of PKL protein. The C-terminal region of PKL was constructed into the vector pGEX-4T-1 being fused with GST (glutathione S-transferase protein) and expressed in Escherichia coli strain BL21 (DE3) (Novagen, USA). The soluble fusion protein was purified and injected into rabbits as antigen. Polyclonal anti-PKL antibodies were purified from rabbit serum using the purified GST-PKL with glutathione Sepharose 4B (Amersham Pharmacia, Canada). 


\section{Western blot analysis of PKL protein}

Arabidopsis tissues were homogenized in an extraction buffer containing $50 \mathrm{mmol} \mathrm{L}^{-1}$ Tris- $\mathrm{HCl}, \mathrm{pH} 7.5,150 \mathrm{mmol} \mathrm{L}^{-1}$ $\mathrm{NaCl}, 10 \mathrm{mmol} \mathrm{L}^{-1} \mathrm{MgCl}_{2}, 0.1 \%$ Nonidet P-40, 1 mmol L ${ }^{-1}$ phenylmethylsulfonyl fluoride, and $1 \times$ complete protease inhibitor (Roche, Switzerland). The extracts were centrifuged twice at $13,000 \times \mathrm{g}$ for $10 \mathrm{~min}$ at $4^{\circ} \mathrm{C}$, and the protein concentration in the supernatant was determined by Bradford assay (Bio-Rad, USA). Protein samples were boiled in sample buffer, run on SDS-PAGE (sodium dodecyl sulfate-poyacrylamide gel electropheresis) gels ( $8 \%$ or $12 \%$ ), and blotted onto polyvinylidene difluoride membranes (Millipore, USA). The blots were probed with a primary polyclonal antibody against PKL.

\section{Total RNA extraction and RT-PCR}

Total RNA was extracted with TRIzol (Invitrogen, USA). After quantification, total RNA was reversely transcribed using the ReverTra Ace qPCR RT kit (TOYOBO, Japan). cDNAs were analyzed by quantitative PCR. A fragment encoding PP2A subunit A3 (At1g13320) was amplified as endogenous control to normalize the relative expression with primers as described previously (Czechowski et al., 2005). At least three independent experiments on independent tissue samples were performed.

\section{RNA-seq and data analyses}

For RNA-seq analysis, total RNA of 3-day-old seedlings, aerial part of 10-day-old seedlings and 25-day-old shoot apices of Col-0 wild-type and $p k l-1$ mutant were extracted with RNeasy Plant Mini Kit (Qiagen, Germany) according to the manufacturer's recommendations. The samples were purified using RNeasyMinElute Cleanup Kit (Qiagen). The quality of all the RNA samples (RNA Integrity Number (RIN) over 6.8) were determined using a 2100 Bioanalyzer (Agilent, USA). The RNA samples were then used to construct libraries and sequenced by 50-cycle single-end sequencing on the HiSeq2000 platform at the Biodynamics Optical Imaging Center, Peking University. Each sample was sequenced in duplicates. All the reads from the sequencing results were cleaned up and aligned to the TAIR10 representative transcriptome using TopHat package. Differentially expressed genes were identified using the Cufflinks package with $P$-value $\leqslant 0.05$ and $Q$-value $\leqslant 0.05$. The differentially expressed genes were classified into many gene ontologies by the program at the agriGO website (http://bioinfo.cau.edu.cn/agriGO/index.php).

\section{ChIP-seq and ChIP-qPCR assays}

Chromatin immunoprecipitation (ChIP) was performed as described previously (Saleh et al., 2008). Briefly, plant materials grown under different conditions were harvested and fixed with $1 \%$ formaldehyde under vacuum for $15 \mathrm{~min}$ at room temperature. The reaction was stopped by adding 2 mol L $\mathrm{L}^{-1}$ Glycine to a final concentration of $100 \mathrm{mmol} \mathrm{L}^{-1}$ and applying vacuum for an additional $5 \mathrm{~min}$ at room temperature. Plant tissues were washed and ground to a fine powder in lipid nitrogen before being resuspended in nucleus lysis buffer, and the nuclear DNA were fragmented into an average size of approximately $500 \mathrm{bp}$ by sonication. After removal of cellular debris, a small aliquot of each sample was stored for reverse crosslinking as a direct total input DNA control. Other chromatin solutions were precleared by incubating with Protein G Dynabeads (Invitrogen) for $1 \mathrm{~h}$ at $4^{\circ} \mathrm{C}$. The Dynabeads were then removed, and immune-precipitation was performed with purified anti-PKL antibody overnight at $4^{\circ} \mathrm{C}$ under constant agitation. An equal amount of sample without antibody was used as a mock control. Freshly prepared Protein G Dyna beads were added into the chromatin solution and incubated for $2 \mathrm{~h}$ at $4^{\circ} \mathrm{C}$ to pull down DNA-protein-antibody complexes. After washing with low-salt wash buffer $\left(150 \mathrm{mmol} \mathrm{L}^{-1} \mathrm{NaCl}\right.$, $0.1 \%$ SDS, $1 \%$ Triton $\mathrm{X}-100,2 \mathrm{mmol} \mathrm{L}^{-1}$ EDTA (ethylenediamine tetraacetic acid), and $20 \mathrm{mmol} \mathrm{L}^{-1}$ Tris-HCl, pH 8.0), high-salt wash buffer $\left(500 \mathrm{mmol} \mathrm{L}^{-1}\right.$ $\mathrm{NaCl}, 0.1 \%$ SDS, $1 \%$ Triton $\mathrm{X}-100,2 \mathrm{mmol} \mathrm{L}^{-1}$ EDTA, and 20 mmol L ${ }^{-1}$ Tris-HCl, pH 8.0), LiCl wash buffer $(0.25 \mathrm{~mol}$ $\mathrm{L}^{-1} \mathrm{LiCl}, 1 \%$ Nonidet $\mathrm{P}-40,1 \%$ sodium deoxycholate, 1

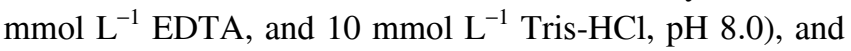
TE buffer $\left(10 \mathrm{mmol} \mathrm{L}^{-1}\right.$ Tris- $\mathrm{HCl}, \mathrm{pH} 8.0$, and $1 \mathrm{mmol} \mathrm{L}^{-1}$ EDTA), the immunocomplexes were eluted from the protein $\mathrm{G}$ Dynabeads. The samples were kept at $65^{\circ} \mathrm{C}$ overnight with $\mathrm{NaCl}$ for reverse crosslinking and then treated with proteinase $\mathrm{K}$ for $1 \mathrm{~h}$ at $45^{\circ} \mathrm{C}$. DNA was extracted with phenol-chloroform and precipitated with ethanol containing one-tenth volume of $3 \mathrm{~mol} \mathrm{~L}^{-1} \mathrm{NaAc}$.

ChIPed DNAs were analyzed by sequencing at the Biodynamics Optical Imaging Center, Peking University, and quantitative PCR (qPCR). Primers were designed to amplify 80- to 150-bp DNA fragments residing in the desired regions of the genes tested (Czechowski et al., 2005). For quantitative PCR, ChIP DNAs were analyzed by Chromo4 (Bio-Rad), in which iQ SYBR Green Supermix (Bio-Rad) was used as the reaction system.

Compliance and ethics The author(s) declare that they have no conflict of interest.

Acknowledgements We thank Dr. Doris Wagner from University of Pennsylvania for generously providing lfy-6 35S::LFY-GR seeds and helpful discussion, Xiaofeng Cao from Institute of Genetics and Development Biology, Chinese Academy of Sciences for helpful discussion and material support, and Carl K.Y. Ng from University College Dublin for language editing and discussion.

Author contributions Liu-Min Fan and Xing Fu conceived and designed the experiments. Xing Fu, Qiang Liang, Chaonan Li and Yangyang Zhou performed the experiments and analyzed the data. Hang He analyzed the RNA-seq data. Xing Fu and Liu-Min Fan wrote the paper. 
Aichinger, E., Villar, C.B., Farrona, S., Reyes, J.C., Hennig, L., and Kohler, C. (2009). CHD3 proteins and polycomb group proteins antagonistically determine cell identity in Arabidopsis. PLoS Genet 5, e1000605.

Balanza, V., Martinez-Fernandez, I., and Ferrandiz, C. (2014). Sequential action of FRUITFULL as a modulator of the activity of the floral regulators SVP and SOC1. J Exp Bot 65, 1193-1203.

Blazquez, M.A., Green, R., Nilsson, O., Sussman, M.R., and Weigel, D. (1998). Gibberellins promote flowering of Arabidopsis by activating the $L E A F Y$ promoter. Plant Cell 10, 791-800.

Blazquez, M.A., Soowal, L.N., Lee, I., and Weigel, D. (1997). LEAFY expression and flower initiation in Arabidopsis. Development 124, 3835-3844.

Bradley, D., Vincent, C., Carpenter, R., and Coen, E. (1996). Pathways for inflorescence and floral induction in Antirrhinum. Development 122, 1535-1544

Czechowski, T., Stitt, M., Altmann, T., Udvardi, M.K., and Scheible, W.R. (2005). Genome-wide identification and testing of superior reference genes for transcript normalization in Arabidopsis. Plant Physiol 139, 5-17.

Eshed, Y., Baum, S.F., and Bowman, J.L. (1999). Distinct mechanisms promote polarity establishment in carpels of Arabidopsis. Cell 99, 199-209.

Ferrandiz, C., Gu, Q., Martienssen, R., and Yanofsky, M.F. (2000). Redundant regulation of meristem identity and plant architecture by FRUITFULL, APETALA1 and CAULIFLOWER. Development 127, 725-734.

Fukaki, H., Taniguchi, N., and Tasaka, M. (2006). PICKLE is required for SOLITARY-ROOT/IAA14-mediated repression of ARF7 and ARF19 activity during Arabidopsis lateral root initiation. Plant J 48, 380-389.

Furuta, K., Kubo, M., Sano, K., Demura, T., Fukuda, H., Liu, Y.G., Shibata, D., and Kakimoto, T. (2011). The CKH2/PKL chromatin remodeling factor negatively regulates cytokinin responses in Arabidopsis calli. Plant Cell Physiol 52, 618-628.

Han, J., and Kamber, M. (2001). Data Mining: Concepts and Techniques. San Francisco: Morgan Kaufmann Publishers.

Harmeyer, K.M., South, P.F., Bishop, B., Ogas, J., and Briggs, S.D. (2015). Immediate chromatin immunoprecipitation and on-bead quantitative PCR analysis: a versatile and rapid ChIP procedure. Nucleic Acids Res 43, e38.

Henderson, J.T., Li, H.C., Rider, S.D., Mordhorst, A.P., Romero-Severson, J., Cheng, J.C., Robey, J., Sung, Z.R., de Vries, S.C., and Ogas, J. (2004). PICKLE acts throughout the plant to repress expression of embryonic traits and may play a role in gibberellin-dependent responses. Plant Physiol 134, 995-1005.

Ho, K.K., Zhang, H., Golden, B.L., and Ogas, J. (2013). PICKLE is a CHD subfamily II ATP-dependent chromatin remodeling factor. Bioch Biophys Acta 1829, 199-210.

Komeda, Y. (2004). Genetic regulation of time to flower in Arabidopsis thaliana. Annu Rev Plant Biol 55, 521-535.

Lee, J., Oh, M., Park, H., and Lee, I. (2008). SOC1 translocated to the nucleus by interaction with AGL24 directly regulates $L E A F Y$. Plant J $55,832-843$.

Liu, C., Chen, H., Er, H.L., Soo, H.M., Kumar, P.P., Han, J.H., Liou, Y.C., and Yu, H. (2008). Direct interaction of AGL24 and SOC1 integrates flowering signals in Arabidopsis. Development 135, 1481-1491.

Michaels, S.D., Ditta, G., Gustafson-Brown, C., Pelaz, S., Yanofsky, M., and Amasino, R.M. (2003). AGL24 acts as a promoter of flowering in Arabidopsis and is positively regulated by vernalization. Plant J 33, 867-874
Nagpal, P., Ellis, C.M., Weber, H., Ploense, S.E., Barkawi, L.S., Guilfoyle, T.J., Hagen, G., Alonso, J.M., Cohen, J.D., Farmer, E.E., Ecker, J.R., and Reed, J.W. (2005). Auxin response factors ARF6 and ARF8 promote jasmonic acid production and flower maturation. Development 132, 4107-4118.

Ogas, J., Cheng, J.C., Sung, Z.R., and Somerville, C. (1997). Cellular differentiation regulated by gibberellin in the Arabidopsis thaliana pickle mutant. Science 277, 91-94.

Ogas, J., Kaufmann, S., Henderson, J., and Somerville, C. (1999). PICKLE is a CHD3 chromatin-remodeling factor that regulates the transition from embryonic to vegetative development in Arabidopsis. Proc Natl Acad Sci USA 96, 13839-13844.

Ori, N., Eshed, Y., Chuck, G., Bowman, J.L., and Hake, S. (2000). Mechanisms that control knox gene expression in the Arabidopsis shoot. Development $127,5523-5532$.

Perruc, E., Kinoshita, N., and Lopez-Molina, L. (2007). The role of chromatin-remodeling factor PKL in balancing osmotic stress responses during Arabidopsis seed germination. Plant J 52, 927-936.

Ramirez, J., and Hagman, J. (2009). The Mi-2/NuRD complex: a critical epigenetic regulator of hematopoietic development, differentiation and cancer. Epigenetics 4, 532-536.

Rider, S.D., Henderson, J.T., Jerome, R.E., Edenberg, H.J., Romero-Severson, J., and Ogas, J. (2003). Coordinate repression of regulators of embryonic identity by PICKLE during germination in Arabidopsis. Plant J 35, 33-43.

Rider, S.D., Jr., Hemm, M.R., Hostetler, H.A., Li, H.C., Chapple, C., and Ogas, J. (2004). Metabolic profiling of the Arabidopsis pkl mutant reveals selective derepression of embryonic traits. Planta 219, 489-499.

Saleh, A., Alvarez-Venegas, R., and Avramova, Z. (2008). An efficient chromatin immunoprecipitation (ChIP) protocol for studying histone modifications in Arabidopsis plants. Nat Protocols 3, 1018-1025.

Wagner, D., and Meyerowitz, E.M. (2002). SPLAYED, a novel SWI/SNF ATPase homolog, controls reproductive development in Arabidopsis. Curr Biol 12, 85-94.

Wagner, D., Sablowski, R.W., and Meyerowitz, E.M. (1999). Transcriptional activation of APETALA1 by LEAFY. Science 285, 582-584.

Weigel, D., Alvarez, J., Smyth, D.R., Yanofsky, M.F., and Meyerowitz, E.M. (1992). LEAFY controls floral meristem identity in Arabidopsis. Cell 69, 843-859.

Weigel, D., and Nilsson, O. (1995). A developmental switch sufficient for flower initiation in diverse plants. Nature 377, 495-500.

Winter, C.M., Austin, R.S., Blanvillain-Baufume, S., Reback, M.A., Monniaux, M., Wu, M.F., Sang, Y., Yamaguchi, A., Yamaguchi, N., Parker, J.E., Parcy, F., Jensen, S.T., Li, H., and Wagner, D. (2011). LEAFY target genes reveal floral regulatory logic, cis motifs, and a link to biotic stimulus response. Dev Cell 20, 430-443.

Xue, Y., Wong, J., Moreno, G.T., Young, M.K., Cote, J., and Wang, W. (1998). NURD, a novel complex with both ATP-dependent chromatin-remodeling and histone deacetylase activities. Mol Cell 2, 851-861.

Yamaguchi, A., Wu, M.F., Yang, L., Wu, G., Poethig, R.S., and Wagner, D. (2009). The microRNA-regulated SBP-Box transcription factor SPL3 is a direct upstream activator of LEAFY, FRUITFULL, and APETALA1. Dev Cell 17, 268-278.

Zhang, H., Bishop, B., Ringenberg, W., Muir, W.M., and Ogas, J. (2012). The CHD3 remodeler PICKLE associates with genes enriched for trimethylation of histone H3 lysine 27. Plant Physiol 159, 418-432.

Zhang, H., Rider, S.D., Jr., Henderson, J.T., Fountain, M., Chuang, K., Kandachar, V., Simons, A., Edenberg, H.J., Romero-Severson, J., Muir, W.M., and Ogas, J. (2008). The CHD3 remodeler PICKLE promotes trimethylation of histone H3 lysine 27. J Biol Chem 283, 22637-22648.

Open Access This article is distributed under the terms of the Creative Commons Attribution License which permits any use, distribution, and reproduction in any medium, provided the original author(s) and source are credited. 


\section{SUPPORTING INFORMATION}

Figure S1 GO annotation of differentially expressed genes in 3-day-old seedlings. AgriGO analysis computes GO term enrichment in one set of genes by comparing it to another set, named the target and reference list, respectively. $P<0.05$, FDR $<0.05$. The darker of the box, the higher the enrichment of the genes in the pathway.

Figure S2 GO annotation of differentially expressed genes in 10-day-old seedlings. AgriGO analysis computes GO term enrichment in one set of genes by comparing it to another set, named the target and reference list, respectively. $P<0.05$, FDR $<0.05$. The darker of the box, the higher the enrichment of the genes in the pathway.

Table S1 Summary of RNA-seq data and mapping statistics

Table S2 A subset of flowering-related genes affected by the $p k l$ mutation

Table S3 $L F Y$ and $L F Y$-regulated genes affected by the $p k l$ mutation

Table S4 Primers used in the experiment shown in Figure 4

Table S5 Primers used in the experiment shown in Figures 5 and 6

The supporting information is available online at life.scichina.com and link.springer.com. The supporting materials are published as submitted, without typesetting or editing. The responsibility for scientific accuracy and content remains entirely with the authors. 